\title{
SMQT-based Tone Mapping Operators for High Dynamic Range Images
}

\author{
Mikael Nilsson ${ }^{1}$ \\ ${ }^{1}$ Centre of Mathematical Sciences, Lund University, Lund, Sweden \\ micken@maths.lth.se
}

Keywords: $\quad$ Tone Mapping Operation; High Dynamic Range; Successive Mean Quantization Transform; Processing Speed

Abstract: In this paper, tone mapping operations based on the nonlinear Successive Mean Quantization Transform (SMQT) are proposed in order to convert high dynamic range images to low dynamic range images. A SMQTbased tone mapping applied on the luminance channel is derived as well as a SMQT-based method working directly on all RGB channels. Both methods are compared to other state-of-the-art methods and produce visually similar results. The processing speeds of the SMQT-based methods are discussed and found to be some of the fastest reported on a single CPU. Furthermore, additional improvement regarding the processing speed and its impact on image quality is investigated.

\section{INTRODUCTION}

The real world scenes we experience in our daily life have a very wide range of luminance values. The human visual system is capable of perceiving scenes over five orders of magnitude and can gradually adapt to scenes with dynamic ranges of over nine orders of magnitude (Duan and Qiu, 2004). While humans can perceive scenes over five orders the current consumer products today typically has display and acquisition technology ranging from two to three orders of magnitude. Hence, pursuing High Dynamic Range (HDR) images is of interest in order to reduce the discrepancy between consumer products and human perception. Recent trends in HDR imaging research involves design of HDR imaging sensors, HDR image generation techniques, encoding methods for efficient transmission and algorithms designed to display HDR images on Low Dynamic Range (LDR) devices (Bandoh et al., 2010; Barakat et al., 2008; Lu et al., 2009). Tone Mapping Operation (TMO) is the process of converting an HDR image to an LDR image. The task of such a TMO needs to address the issue of finding a good balance between emphasizing all features in the image as well as presenting a good contrast while producing the LDR image. Several recent proposed TMOs can be found in the literature (Chen et al., 2005; Duan and Qiu, 2004; Liu et al., 2010; Lee et al., 2010; Qiu et al., 2010; Kuang et al., 2007; Meylan and Susstrunk, 2006; Reinhard et al., 2002; Reinhard and Devlin, 2005; Mantiuk et al., 2008; Durand and Dorsey, 2002; Drago et al., 2003; Pattanaik et al., 2000).

TMOs can generally be divided into two broad categories: global and local tone mapping (Reinhard et al., 2005). Global tone mapping techniques utilize a single, and typically highly nonlinear, spatially invariant mapping function. In many cases the nonlinear functions are combinations of logarithmic and/or exponential operators (Reinhard et al., 2002; Drago et al., 2003; Reinhard, 2011). Global TMOs tend to preserve the subjective perception of the scene and have the advantage of being simple and fast. However, global processing may cause a loss of contrast, which is apparent in the loss of detail visibility (Zhang and Kamata, 2008). Local TMOs utilize local neighborhood around a pixel in the mapping. This implies that two different HDR image pixels with the same intensity can be mapped to different LDR image values. This can make the local TMOs bring out more details compared to a global method, but usually at a cost of longer processing time. However, local methods may also cause "halo" effects or ringing artifacts in the resulting LDR image (Reinhard et al., 2002). The processing time of a TMO is also an issue; several of the existing methods are computationally expensive. Hence, utilizing Graphics Processing Unit (GPU) (Zhao et al., 2008) or design of hardware architecture (Wang et al., 2007) for existing methods are of interest unless the TMO method used can run at acceptable times on a single CPU.

In this paper, new global TMOs are proposed utilizing the nonlinear Successive Mean Quantization Transform (SMQT) (Nilsson et al., 2005b; Nilsson 
et al., 2005a). To the best of the author knowledge no prior tone mapping operation of high dynamic range images based on the SMQT has been proposed or investigated. The paper will show that the SMQT is a good choice for mapping HDR images to LDR. In particular, it will be shown that the method is fast, and gives reasonable visual results comparable to the state-of-the-art.

Two versions of the tone mapping operations are considered here, one working on luminance and one version working directly on all RGB channels. The framework of the luminance approach is in line with other works (but they do not use or propose the use of the SMQT). The presented all channel approach is to the best of the author knowledge novel. It should be emphasized that, in order to get a general framework, the derivation, experiments and processing speeds are conducted using numbers in double precision describing the HDR image. Hence, if RGB histograms of the HDR image is available, the processing described could be performed on those histograms and lead to further speed improvements.

The paper is organized as follows. In the next section formal description of the SMQT is revisited. Section 3 presents how the SMQT TMO works on luminance values as well as the all channel RGB image and makes a comparison. In section 4 the processing speed is investigated and a comparison to other stateof-the-art TMOs is performed. Finally, conclusions are presented.

\section{DESCRIPTION OF THE SMQT}

In general, a data point $x$ in a set $\mathcal{D}$ consisting of $|\mathcal{D}|=D$ data points are the input to the transform. The value of a data point is denoted by $\mathbf{V}(x)$. Note that the data structure can be arbitrary, that is $\mathcal{D}$ can be a vector, a matrix or some arbitrary form. In this paper, the set $\mathcal{D}$ is a HDR image of size $D=M \times N$ if gray-scale (luminance) and $D=M \times N \times 3$ if color.

The SMQT has only one parameter input: the level $L$ (indirectly, it will also have the number of data points $D$ as an important input). The output set from the transform is denoted $\mathcal{M}$. It has the same form as the input, that is $\mathcal{D}$ could be a color HDR image of size $M \times N \times 3$ then $\mathcal{M}$ is also a color image of size $M \times N \times 3$ but with different range of values. The transform of level $L$ from $\mathcal{D}$ to $\mathcal{M}$ is denoted

$$
\mathrm{SMQT}_{L}: \mathcal{D} \rightarrow \mathcal{M} .
$$

The $\mathrm{SMQT}_{L}$ function can be described by a binary tree where the vertices are Mean Quantization Units
(MQUs). A MQU consists of three steps: a mean calculation, a quantization and a split of the input set.

The first step of the MQU finds the mean of the data, denoted $\overline{\mathbf{V}}$, according to

$$
\overline{\mathbf{V}}=\frac{1}{|\mathcal{D}|} \sum_{x \in \mathcal{D}} \mathbf{V}(x)
$$

The second step uses the mean to quantize the values of data points into $\{0,1\}$. A comparison function is defined as

$$
\xi(\mathbf{V}(x), \overline{\mathbf{V}})= \begin{cases}1, & \text { if } \mathbf{V}(x)>\overline{\mathbf{V}} \\ 0, & \text { else. }\end{cases}
$$

If $\otimes$ denotes concatenation, then

$$
\mathcal{U}=\bigotimes_{x \in \mathcal{D}} \xi(\mathbf{V}(x), \overline{\mathbf{V}})
$$

is the mean quantized set. The set $\mathcal{U}$ is the main output from a MQU. The third step splits the input set into two subsets:

$$
\begin{aligned}
& \mathcal{D}_{0}=\{x \mid \mathbf{V}(x) \leq \overline{\mathbf{V}}, \forall x \in \mathcal{D}\} \\
& \mathcal{D}_{1}=\{x \mid \mathbf{V}(x)>\overline{\mathbf{V}}, \forall x \in \mathcal{D}\},
\end{aligned}
$$

where $\mathcal{D}_{0}$ propagates the left and $\mathcal{D}_{1}$ propagates the right of the binary tree, see Fig. 1.

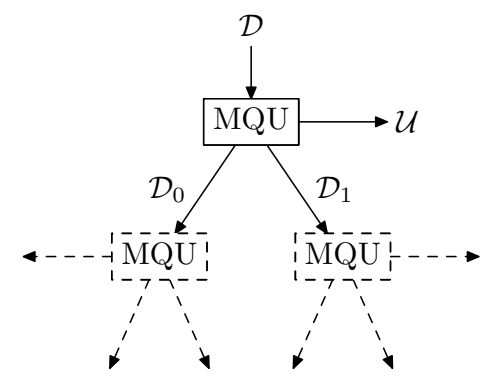

Figure 1: The operation of one Mean Quantization Unit (MQU).

The output set $\mathcal{U}$ from a MQU is not a value or a similarity coefficient as in the linear transforms. Instead, $\mathcal{U}$ can be interpreted as the structure of $\mathcal{D}$. Hence, the MQU is independent of the gain and bias adjustments of the input.

The MQU constitutes the main computing unit for the SMQT. The first level transform, $\mathrm{SMQT}_{1}$, is based on the output from a single MQU, where $\mathcal{U}$ is the output set at the root node. The outputs in the binary tree need extended notation. The output set from one MQU in the tree is denoted $\mathcal{U}_{(l, n)}$, where $l=$ $1,2, \ldots, L$ is the current level, and $n=1,2, \ldots, 2^{(l-1)}$ is the output number for the MQU at level $l$, see Fig. 2.

Weighting of the values of the data points in the $\mathcal{U}_{(l, n)}$ sets is performed and the final $\mathrm{SMQT}_{L}$ is found 


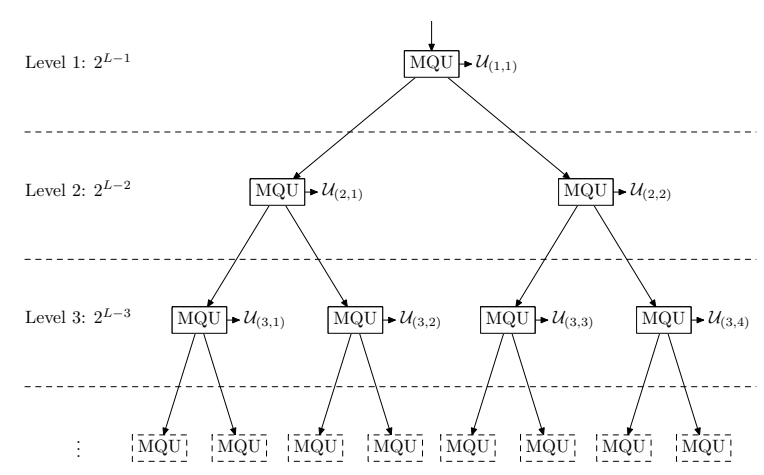

Figure 2: The Successive Mean Quantization Transform (SMQT) as a binary tree of Mean Quantization Units (MQUs).

by adding the results. The weighting is performed by $2^{L-l}$ at each level $l$. Hence, the result for the $\mathrm{SMQT}_{L}$ can be found in the following way

$$
\begin{aligned}
\mathcal{M}= & \left\{x \mid \mathbf{V}(x)=\sum_{l=1}^{L} \sum_{n=1}^{2^{l-1}} \mathbf{V}\left(u_{(l, n)}\right) \cdot 2^{L-l},\right. \\
& \left.\forall x \in \mathcal{D}, \forall u_{(l, n)} \in \mathcal{U}_{(l, n)}\right\}
\end{aligned}
$$

As a consequence of this weighing, the number of quantization levels, denoted by $Q_{L}$, for a structure of level $L$ will be $Q_{L}=2^{L}$. Since the main aim here is to produce low dynamic range images the most natural choice is $L=8$.

\section{SMQT-BASED TONE MAPPING OPERATIONS}

Most color imaging systems today use a single image sensor and a Color Filter Array (CFA) to capture full color images. A color filter array enables each pixel to capture the intensity of light with different color spectrum. The most common design of CFA is the GRGB Bayer pattern (Bayer, 1976) which consists of two greens, one red, and one blue component. A CFA image is then interpolated via a demosaicing algorithm and produces a final RGB color image. Hence, in most cases color image are initially in RGB format from which the proposed TMOs are derived.

Let a RGB HDR image be denoted $I_{H D R}(i, j, k)$ where $i=0,1, \ldots, M-1$ is the row index, $j=$ $0,1, \ldots, N-1$ the column index and $k=0,1,2$ the color channels red, green and blue. Additionally, let $R_{H D R}(i, j)=I_{H D R}(i, j, 0), \forall i, j, G_{H D R}(i, j)=$ $I_{H D R}(i, j, 1), \forall i, j$ and $B_{H D R}(i, j)=I_{H D R}(i, j, 2), \forall i, j$ be the RGB matrices for the HDR image. Same notations will follow for a LDR image, but with subscript
LDR. To simplify notation, the indices $i, j$ and $k$ are dropped unless specifically required.

In general, a TMO can be seen as a 3-D matrix mapping

$$
I_{L D R}=f\left(I_{H D R}\right)
$$

where $f(\bullet)$ is the general tone mapping. One approach commonly adopted for tone mapping is to convert the RGB to luminance, $L_{H D R}$, as

$$
L_{H D R}=0.299 R_{H D R}+0.587 G_{H D R}+0.114 B_{H D R}
$$

and perform a mapping $L_{L D R}=g\left(L_{H D R}\right)$ on the luminance only. To find the LDR color channels, the desaturated color-to-luminance ratios can be used (Schlick, 1994; Mantiuk et al., 2009)

$$
\begin{aligned}
& R_{L D R}=\left(\frac{R_{H D R}}{L_{H D R}}\right)^{s} L_{L D R} \\
& G_{L D R}=\left(\frac{B_{H D R}}{L_{H D R}}\right)^{s} L_{L D R} \\
& B_{L D R}=\left(\frac{G_{H D R}}{L_{H D R}}\right)^{s} L_{L D R}
\end{aligned}
$$

where $s$ controls color saturation. Other techniques may be employed in order to correct the color result (Mantiuk et al., 2009). Note that TMO utilizing the mapping $g(\cdot)$ rely on luminance only, implying color space conversion and that color information is later adjusted to achieve the final result.

Given an HDR image in red, green and blue format, $I_{R G B}$, a TMO aims at finding a good balance between image features, contrast and color fidelity. The SMQT has been shown to have desirable properties in achieving a balanced contrast enhancement for LDR gray-scale images (Nilsson et al., 2005b; Nilsson et al., 2005a). Note that the SMQT can be seen as a nonlinear compression of data points with a higher number of bits to a defined new, and lower, quantization level $L$ set by the user. Hence, the SMQT can be used directly on gray-scale HDR images and one possibility for HDR tone mapping is to apply it in luminance space, see Fig. 3. However, a different

$I_{H D R} \longrightarrow I_{H D R}^{\gamma} \longrightarrow$ Eq. (8) $\longrightarrow L_{H D R} \rightarrow S M Q T_{L}(\cdot) \rightarrow$ Eq. (9) $\longrightarrow I_{L D R}$ Figure 3: HDR to LDR image using the SMQT on luminance channel as a tone mapping operator.

approach is also proposed here, the full RGB image (all channels) will be used to find a mapping function $h(\cdot)$ using the SMQT which then is employed on the channels independently as $R_{L D R}=h\left(R_{H D R}\right)$, $G_{L D R}=h\left(G_{H D R}\right)$ and $B_{L D R}=h\left(B_{H D R}\right)$. Thus, no color space transformation needs to be employed (if given an image already in RGB), see Fig. 4.

An exemplified comparison between the luminance version and all channel RGB SMQT-based 


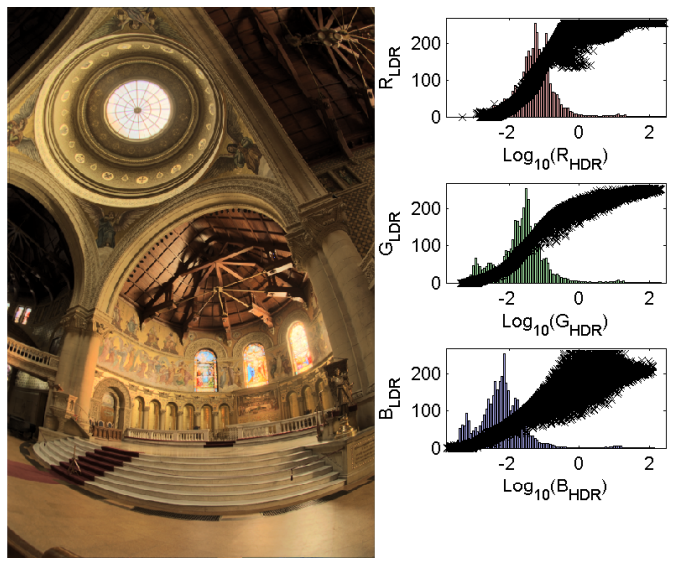

(a) Tone mapping result by SMQT on luminance.

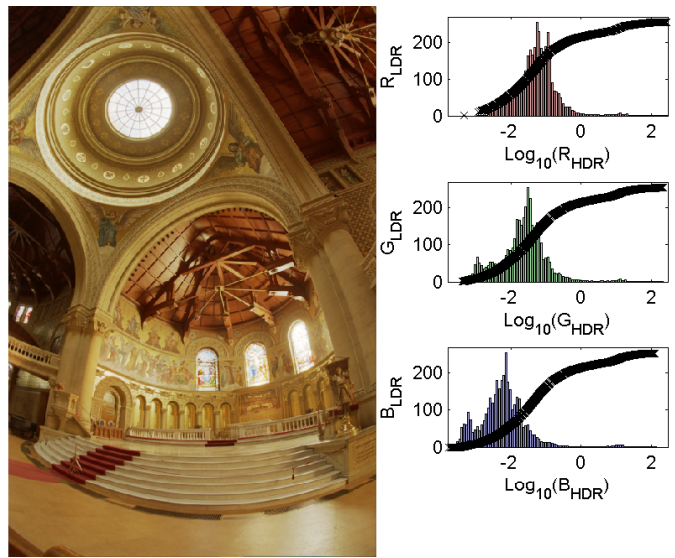

(b) Tone mapping result by SMQT over all RGB channels.

Figure 5: Comparison between luminance SMQT TMO, processing as in Fig. 3, and all RGB channel SMQT TMO, processing as in Fig. 4. The parameters used are $L=8, \gamma=1 / 2.2$ and $s=0.8$.

$$
I_{H D R} \longrightarrow I_{H D R}^{\gamma} \longrightarrow S M Q T_{L}(\cdot) \longrightarrow I_{L D R}
$$

Figure 4: HDR to LDR image using the SMQT on all RGB channels as a tone mapping operator.

TMO can be found in Fig. 5, to the right of each image the log-luminance of the HDR intensity is plotted versus the mapped intensity of the LDR image for each color channel. Note that a property following from the SMQT applied on all channel RGB, as well as the luminance mapping according to Eq. 9, is that the order of intensity values in $\mathrm{R}, \mathrm{G}$ and $\mathrm{B}$ for a pixel will be the same in input and output since the channels use the same monotone mapping, see Fig. 5.

\section{EXPERIMENTAL RESULTS}

The proposed luminance SMQT and all channel RGB SMQT based TMO are implemented in Matlab ${ }^{\circledR}$, with parts of the SMQT operation rewritten in ANSI $\mathrm{C}$ and wrapped to a mex file in order to gain speed. The processing speed for the processing chains found in Fig. 3 and Fig. 4 (note that it includes gamma correction) for HDR images of various size can be found in Fig.6. The processing speed between the luminance and all channel RGB method are very similar, but the all channel RGB is somewhat faster. This implies that SMQT on $M \times N \times 3$ data points is similar to SMQT on $M \times N$ data points with additional color to luminance found in Eq. 8 and producing color output found in Eq. 9. Hence, if the SMQT processing speed can be reduced the all channel RGB TMO is expected to increase its FPS more compared to the luminance version. For a timing comparison with other work, Mantiuk et al. implemented a TMO in Matlab and re-

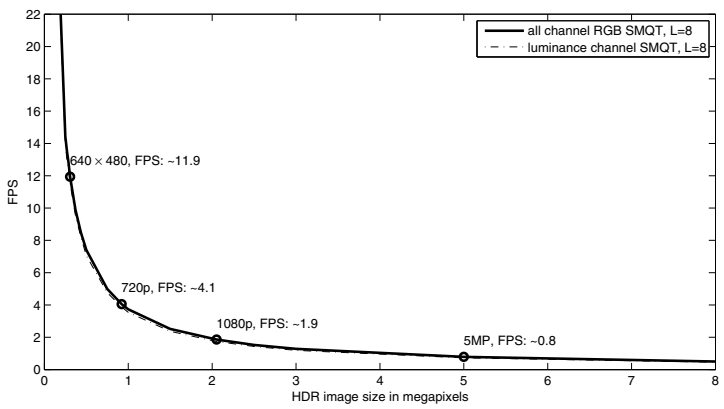

Figure 6: FPS for different sizes of HDR images. Circles with text indicate FPS for the SMQT-based all channel RGB TMO.

ported a processing speed of 1.7 seconds ( 0.59 FPS) on $2.6 \mathrm{GHz}$ CPU for a $1 \mathrm{M}$-pixel image (Mantiuk et al., 2008). The proposed luminance SMQT-based TMO achieves 3.60 FPS and the all channel RGB SMQTbased TMO 3.80 FPS on a $2.13 \mathrm{GHz}$ CPU for a $1 \mathrm{M}$ pixel image. However, GPU and hardware architecture solutions of TMOs naturally report higher frame rates (Zhao et al., 2008; Wang et al., 2007). A visual comparison to other state-of-the-art TMOs can be found in Fig. 7. The SMQT-based methods use the parameters $L=8, \gamma=1 / 2.2$ and $s=0.8$ and the other TMOs use the default parameters defined in the Luminance HDR software ${ }^{1}$. Note that the SMQT-based TMOs, similar to other state-of-the-art TMOs, produce naturally looking LDR images.

\footnotetext{
${ }^{1}$ Luminance HDR version 2.2.1 from http://qtpfsgui.sourceforge.net/
} 


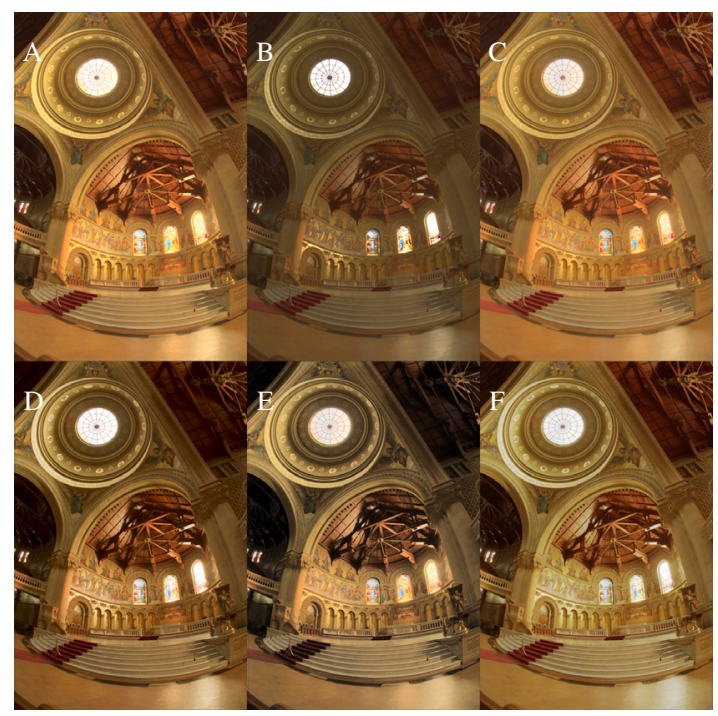

(a) HDR image Stanford memorial church.

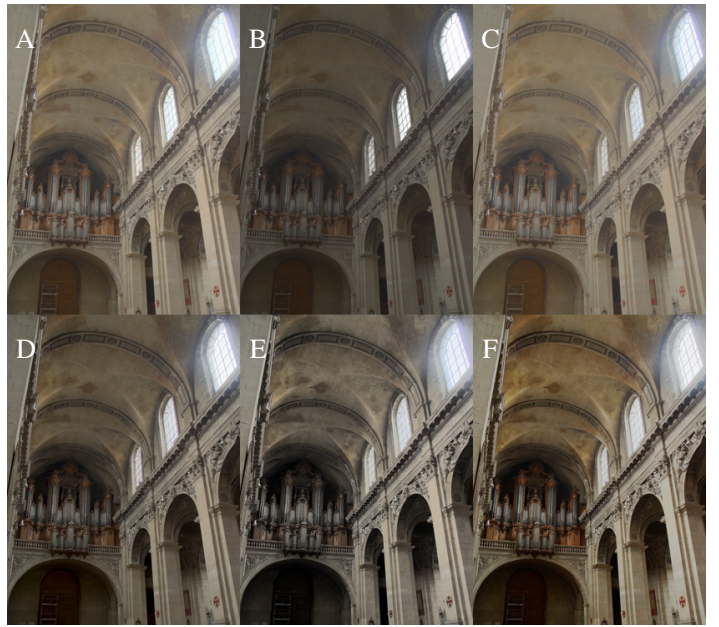

(b) HDR image Nancy church 2 from MPI.

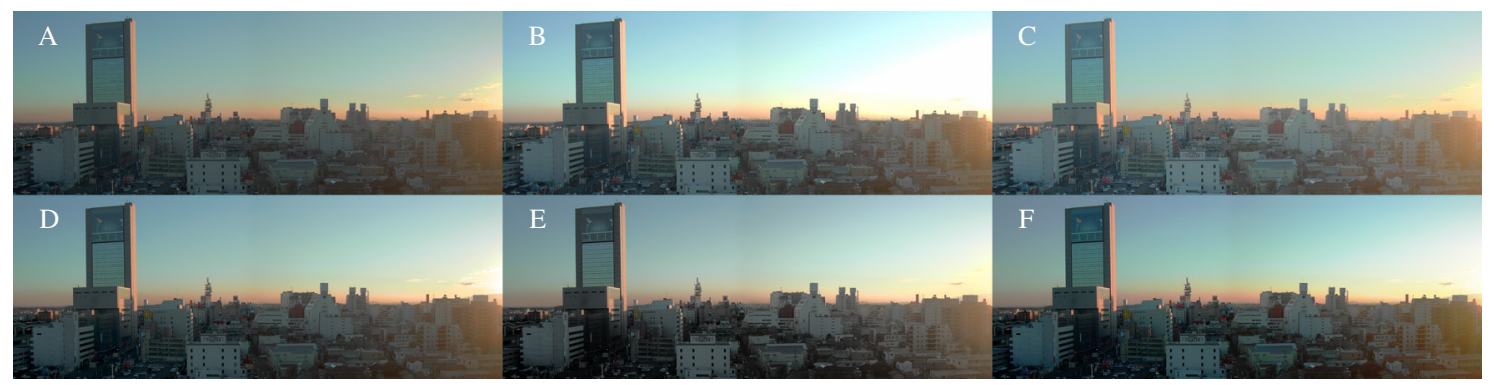

(c) HDR image Iwate from MPI.
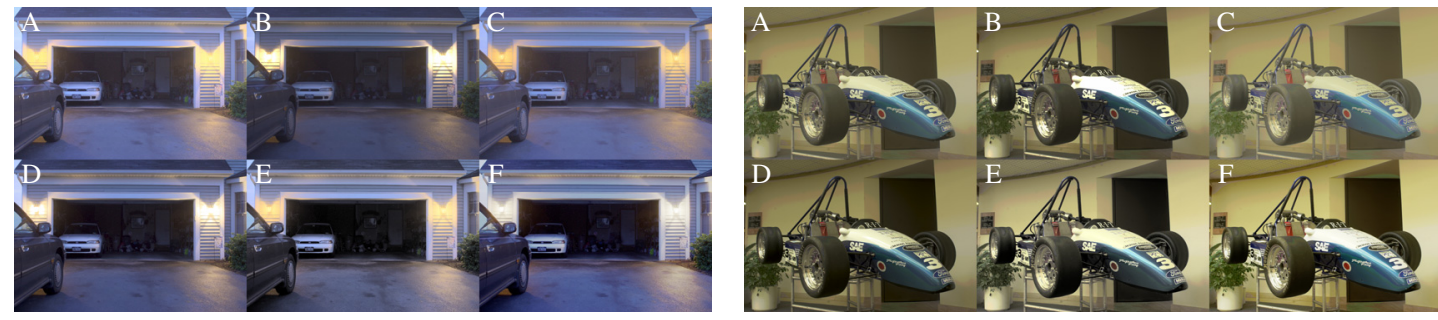

(d) HDR image Garage from RIT MCSL High Dynamic (e) HDR image Racecar from RIT MCSL High Dynamic Range Image Database.

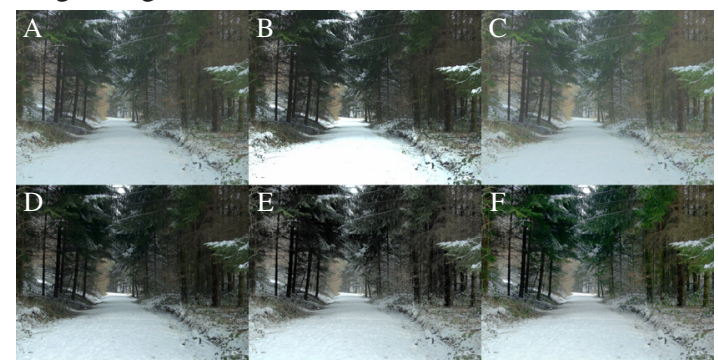

(f) HDR image Snow from MPI. Range Image Database.

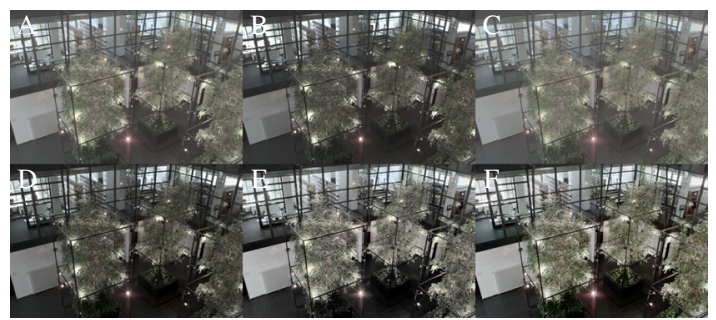

(g) HDR image MPI Atrium 1 from MPI.

Figure 7: Tone mapped HDR images. TMO by (A) Reinhard et al. (Reinhard et al., 2002), (B) Durand and Dorsey (Durand and Dorsey, 2002), (C) Drago et al. (Drago et al., 2003), (D) Mantiuk et al. (Mantiuk et al., 2008), (E) the proposed luminance channel SMQT-based method and (F) the proposed all channel RGB SMQT-based method. Zooming in the pdf images is recommended for detail studies. 


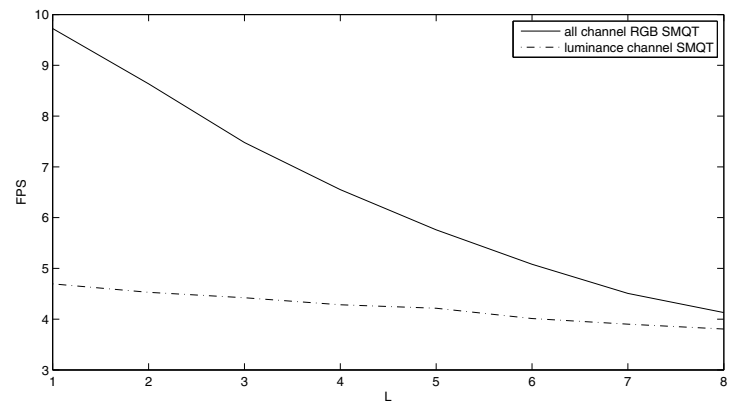

Figure 8: Processing speeds on a one megapixel image in Frames Per Second (FPS) on a $2.13 \mathrm{GHz}$ CPU. The parameters used are $\gamma=1 / 2.2$ and $s=0.8$.

\subsection{Improving Processing Speed}

While the processing speed for the proposed SMQT TMOs are some of the fastest reported on a single CPU, with regard to implementation on embedded systems, it is still desirable to achieve better processing speeds. The choice of the level used so far is $L=8$, simply due to the fact that the aim is eight bit images. However, selecting a level $L$ lower can be a direct way to reduce processing speed but with reduced image quality. Note that the final output is still desired to be eight bits and selecting $L$ as a lower number results in $L$ bit output. Hence, a direct way to again produce a eight bit result from any selected $L$ is to multiply the result from Fig. 3 or Fig. 4 with $2^{8-L}$ resulting in an eight bit image

$$
I_{8 \text { bit } L D R}=I_{L D R} \cdot 2^{8-L} \text {. }
$$

As mentioned, it is expected that the all channel RGB will benefit more than the luminance version in a reduction of the SMQT processing speed, see Fig. 8. The all channel RGB method benefits more from the reduction in processing speed of the SMQT, for example at $L=5$ the speed is more than $30 \%$ higher in FPS compared to the luminance method on a one megapixel image.

Naturally, the image quality decreases as the level is decreased, see Fig. 9. A subjective observation from the resulting images constructed with different levels is that around $L=4$ and $L=5$ acceptable results can be achieved. In order to objectify this statement the Peak Signal to Noise Ratio (PSNR) is calculated for the luminance of images and as reference the eight bit image is used. The seven images found in Fig. 7 are used and the mean PSNR is calculated, see Fig.11(a). Selecting a threshold at $30 \mathrm{~dB}$, which has been found to be suitable from a psychophysical viewpoint (F. Xiao, 2005), coincide with choosing a level around four for both methods. Additionally, the quality mapped to mean opinion scores, $Q_{M O S}$, from
HDR-VDP-2 (Mantiuk et al., 2011) is investigated. The settings for $Q_{M O S}$ calculation are color encoding selected as $s R G B$-display and pixels-per-degree as 30 . The seven images found in Fig. 7 are used and the mean $Q_{M O S}$ is calculated, see Fig.11(b). Note that around $L=5$ the $Q_{M O S}$ starts dropping and PSNR drops below $30 \mathrm{db}$ at around $L=4$, hence this coincides well with the initial subjective statement from observing Stanford memorial, see Fig. 9. Furthermore, the all channel RGB SMQT keeps $Q_{M O S}$ higher compared to luminance channel SMQT at $L<5$. Hence, a reasonable conclusion from this analysis is that using $L=5$ could be a fair choice in order to reduce computation speed while retaining decent image quality. A comparison of processing speed using $L=5$ can be found in Fig. 10 .

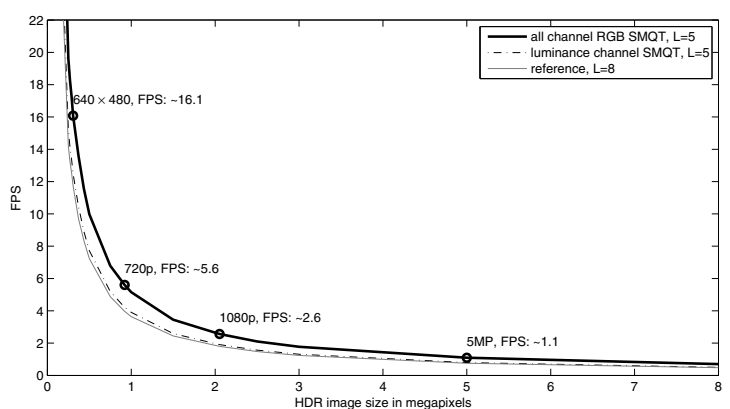

Figure 10: FPS for different sizes of HDR images and $\mathrm{L}=5$. Reference is average FPS from the two curves found in Fig. 6. Circles with text indicate FPS for the SMQT-based all channel RGB TMO.

\section{CONCLUSIONS}

Two novel global tone mapping operations based on the nonlinear Successive Mean Quantization transform (SMQT) have been proposed and investigated. One method is based on applying the SMQT on luminance channel and another is based on performing the SMQT on all RGB channels. The methods were compared to other state-of-the-art methods and produce visually similar results. Regarding the processing speed, they are some of the fastest reported on a single CPU and can run close to 12 FPS on VGA $(640 \times 480)$ with a $2.13 \mathrm{GHz}$ computer in default operation. The methods were further investigated and it was demonstrated that the all channel RGB has more desirable properties compared to the luminance method considering processing speed reduction and associated image degradation. It is found, using PSNR and $Q_{M O S}$, that a reasonable image degradation which allows speedup can be achieved at the choice of level $L=5$ in the SMQT. Utilizing $L=5$ compared 


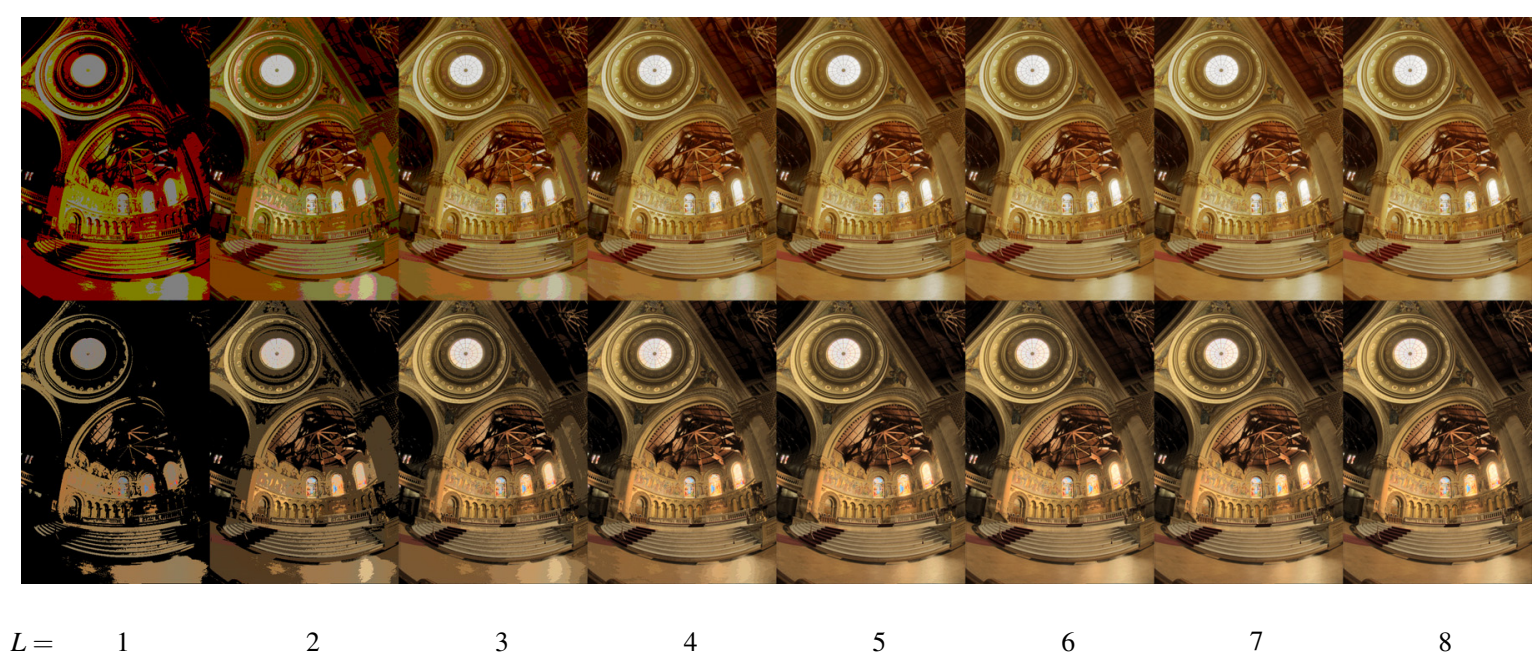

Figure 9: Example of image quality on Stanford memorial for different levels $L$. Top is SMQT-based all channel RGB tone mapping operation. Bottom is SMQT-based luminance channel tone mapping operation.

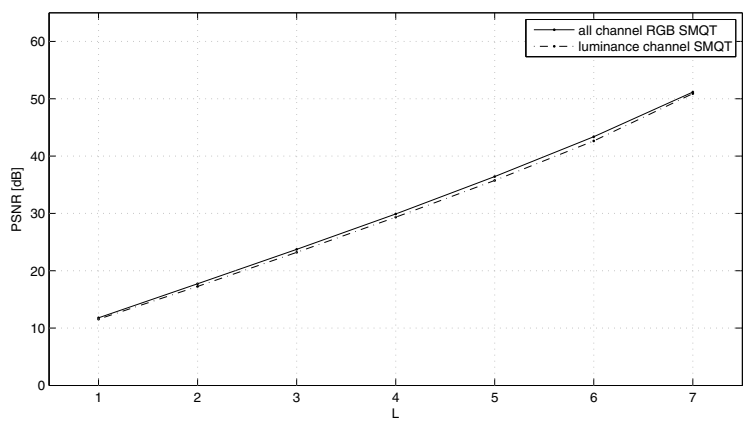

(a) PSNR for different levels using level eight as reference.

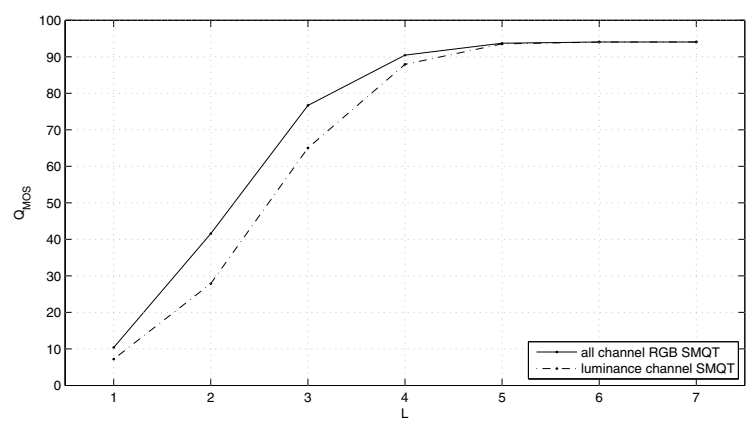

(b) $Q_{M O S}$ quality measure for different levels using level eight as reference.

Figure 11: Peak Signal to Noise Ration (PSNR) and quality measure $\left(Q_{M O S}\right)$ for different choices of level $L$. The parameters used are $\gamma=1 / 2.2$ and $s=0.8$. 
to the default operation, the all channel RGB method can run at around 16 FPS while the luminance channel method achieves 12.5 FPS, on a VGA size image and a $2.13 \mathrm{GHz}$ computer.

\section{REFERENCES}

Bandoh, Y., Qiu, G., Okuda, M., Daly, S., Aach, T., and Au, O. (2010). Recent advances in high dynamic range imaging technology. In IEEE International Conference on Image Processing (ICIP), pages $3125-3128$.

Barakat, N., Hone, A., and Darcie, T. (2008). Minimalbracketing sets for high-dynamic-range image capture. IEEE Transactions on Image Processing, 17(10): $1864-1875$.

Bayer, B. E. (1976). Color imaging array. Rochester, NY: Eastman Kodak Company, U.S. Patent 3971065.

Chen, H.-T., Liu, T.-L., and Chang, T.-L. (2005). Tone reproduction: a perspective from luminance-driven perceptual grouping. In IEEE Conference on Computer Vision and Pattern Recognition (CVPR), volume 2, pages $369-376$ vol. 2.

Drago, F., Myszkowski, K., Annen, T., and Chiba, N. (2003). Adaptive logarithmic mapping for displaying high contrast scenes. Computer Graphics Forum, 22:419-426.

Duan, J. and Qiu, G. (2004). Fast tone mapping for high dynamic range images. In International Conference on Pattern Recognition (ICPR), volume 2, pages 847 $-850 \mathrm{Vol} .2$.

Durand, F. and Dorsey, J. (2002). Fast bilateral filtering for the display of high-dynamic-range images. $A C M$ Trans. Graph., 21:257-266.

F. Xiao, J. E. Farrell, B. W. (2005). Psychophysical thresholds and digital camera sensitivity: The thousand photon limit. In Proceedings of the SPIE, volume 5678, pages $75-84$.

Kuang, J., Yamaguchi, H., Liu, C., Johnson, G. M., and Fairchild, M. D. (2007). Evaluating hdr rendering algorithms. ACM Trans. Appl. Percept., 4.

Lee, J. W., Park, R.-H., and Chang, S. (2010). Tone mapping using color correction function and image decomposition in high dynamic range imaging. IEEE Transactions on Consumer Electronics, 56(4):2772 2780.

Liu, C. H., Au, O., Cheng, C. H., and Yip, K. Y. (2010). Two-level optimized tone mapping for high dynamic range images. In IEEE International Conference on Image Processing (ICIP), pages 3153-3156.

Lu, P.-Y., Huang, T.-H., Wu, M.-S., Cheng, Y.-T., and Chuang, Y.-Y. (2009). High dynamic range image reconstruction from hand-held cameras. In IEEE Conference on Computer Vision and Pattern Recognition (CVPR), pages 509-516.

Mantiuk, R., Daly, S., and Kerofsky, L. (2008). Display adaptive tone mapping. ACM Trans. Graph., 27(68):68:1-68:10.
Mantiuk, R., Kim, K. J., Rempel, A. G., and Heidrich, W. (2011). Hdr-vdp-2: a calibrated visual metric for visibility and quality predictions in all luminance conditions. ACM Trans. Graph., 30(4):40.

Mantiuk, R., Mantiuk, R., Tomaszewska, A. M., and Heidrich, W. (2009). Color correction for tone mapping. Comput. Graph. Forum, 28(2):193-202.

Meylan, L. and Susstrunk, S. (2006). High dynamic range image rendering with a retinex-based adaptive filter. IEEE Transactions on Image Processing, 15(9):2820 -2830 .

Nilsson, M., Dahl, M., and Claesson, I. (2005a). Gray-scale image enhancement using the SMQT. In IEEE International Conference on Image Processing (ICIP), volume 1, pages 933-936.

Nilsson, M., Dahl, M., and Claesson, I. (2005b). The successive mean quantization transform. In IEEE International Conference on Acoustics, Speech, and Signal Processing (ICASSP), volume 4, pages 429-432.

Pattanaik, S. N., Tumblin, J., Yee, H., and Greenberg, D. P. (2000). Time-dependent visual adaptation for fast realistic image display. In Proceedings of the 27th annual conference on Computer graphics and interactive techniques, SIGGRAPH, pages 47-54.

Qiu, G., Mei, Y., Lam, K. M., and Qiu, M. (2010). Tone mapping hdr images using optimization: A general framework. In IEEE International Conference on Image Processing (ICIP), pages 3129 -3132.

Reinhard, E. (2011). Tone reproduction and color appearance modeling: Two sides of the same coin? In 19th Color and Imaging Conference.

Reinhard, E. and Devlin, K. (2005). Dynamic range reduction inspired by photoreceptor physiology. IEEE Transactions on Visualization and Computer Graphics, 11(1):13-24.

Reinhard, E., Stark, M., Shirley, P., and Ferwerda, J. (2002). Photographic tone reproduction for digital images. ACM Transactions on Graphics, 21(3):267-276.

Reinhard, E., Ward, G., Pattanaik, S., and Debevec, P. (2005). High Dynamic Range Imaging: Acquisition, Display, and Image-Based Lighting. Morgan Kaufmann Publishers Inc., San Francisco, CA, USA.

Schlick, C. (1994). Quantization techniques for visualization of high dynamic range pictures. In Photorealistic Rendering Techniques, pages 7-20. Springer-Verlag.

Wang, T.-H., Wong, W.-S., Chen, F.-C., and Chiu, C.T. (2007). Design and implementation of a realtime global tone mapping processor for high dynamic range video. In IEEE International Conference on Image Processing (ICIP), volume 6, pages VI -209-VI -212 .

Zhang, J. and Kamata, S.-i. (2008). Adaptive local contrast enhancement for the visualization of high dynamic range images. In International Conference on Pattern Recognition (ICPR), pages $1-4$.

Zhao, H., Jin, X., and Shen, J. (2008). Real-time tone mapping for high-resolution hdr images. In International Conference on Cyberworlds, pages 256-262. 